\title{
Preconditioning elastic full waveform inversion by scattering theory
}

\author{
Keisuke TERANISHI $^{1,2}$, Hitoshi MIKADA ${ }^{1}$ and Junichi TAKEKAWA ${ }^{1}$ \\ ${ }^{1}$ Dept. of Civil and Earth Res. Eng., Kyoto University \\ ${ }^{2}$ Now, Japan Petroleum Exploration Co., Ltd.
}

\begin{abstract}
The waveform analysis is a powerful tool to investigate the physical properties in the areas of interest. Since the wave propagation is influenced by all elastic parameters, it is necessary to include these parameters in the inversion. On the other hand, multi-parameter FWI is a challenging problem because some elastic parameters increases the dimension of the solution space, in other words desensitization of each parameter occurs due to the difference of each radiation pattern and geometrical spreading. Some previous works used preconditioning gradient method using approximate Hessian that takes radiation pattern and geometrical spreading into account in order to compensate the desensitization. However, such methods solve many forward calculations to make the preconditioning operator so the computational cost becomes expensive. In this paper, we suggest new preconditioning gradient method that seeks preconditioning operator by scattering theory instead of many forward calculation to compensate the desensitization. We conduct numerical experiments to compare the results obtained by the new method with those from a conventional method. This method can estimate more reliable results than conventional one and restrain the rise of computational costs.
\end{abstract}

\section{INTRODUCTION}

The importance to use waveform is widely recognized in seismic explorations. Especially, seismic full waveform inversion (FWI) can detect the high-resolution physical properties in the subsurface. Since the seismic wave propagation is the phenomena as a function of kinematic parameters of the medium including density, it is necessary to include all these three parameters in the modeling and inversion (Vireux and Operto, 2009). However, multi-parameter FWI is a challenging problem because parameters are coupled with each other, and the coupling effects prevent the appropriate estimation of the elastic parameters and increase the dimension of the solution space. Especially, the estimation of density is a very difficult exercise because plural elastic parameters including density increases the dimension of the solution space so that any minimization could be trapped in local minima.

Therefore, the density structure is typically estimated by empirical formulas such as the Gardner's relationship (Gardner et al., 1974) or is fixed to a constant value during inversion process. However, the Gardner's relationship could not always show the exact relation between $\mathrm{Vp}$ and density, for example salt domes which have attracted the interest of many researchers in recent years. So this kind of treatment of the density structure could degrade the inversion results.

Since the density parameter is directly included in the elastic wave equation, it is necessary to invert directly. Moreover, some previous works used preconditioning gradient method using approximate Hessian that takes radiation pattern and geometrical spreading into account in order to compensate the desensitization. However, such methods solve many forward calculations so the computational cost becomes expensive. We suggest a new preconditioning gradient method that seeks preconditioning operator by scattering theory instead of many forward calculations.

We incorporate a preconditioning operator of each parameter including Lame constants and density based on the scattering theory into 2D frequency domain FWI. Our study presents the new preconditioning gradient method for FWI based on elastic scattering theory instead of many forward calculations to make the Hessian of each parameter.

\section{THEORY}

A new inversion strategy was built in 1980s known as full-waveform inversion. FWI updates the properties of the subsurface parameter from observed data. To calculate the model parameter direction, waveform inversion algorithm seeks to minimize the residual between the forward 
simulation and the observed seismic data. We define the misfit function as L2 norm as blow.

$$
E=\frac{1}{2} \sum_{s} \int d \omega \sum_{r} \delta u_{i}\left(x_{r}, \omega, x_{s}\right) \delta u_{i}^{*}\left(x_{r}, \omega, x_{s}\right)
$$

where $i$ represents the space direction of displacement, $s$ and $r$ are represented source and receiver respectively, and $\delta u_{i}\left(x_{r}, \omega, x_{s}\right)$ is the residual spectrum. ${ }^{*}$ means complex conjugate.

$$
\delta u_{i}\left(x_{r}, \omega, x_{s}\right)=u_{i}^{o b s}\left(x_{r}, \omega, x_{s}\right)-u_{i}^{c a l}\left(x_{r}, \omega, x_{s}\right)
$$

Our purpose is to estimate the optimal model which minimize the misfit function $\mathbf{E}$.

The gradient method is obtained the gradient which minimizes the $\mathbf{E}$ by updating model parameter.

$$
m_{k+1}=m_{k}-\alpha_{k} H^{-1} \Delta m_{k}
$$

where $m_{k}, \alpha_{k}, \Delta m_{k}$ represents model parameter, step length and modified gradient using conjugate gradient method in iteration $k$, respectively. $H^{-1}$ means preconditioning operator as mentioned below. In conventional gradient method, $H^{-1}$ represents a diagonal matrix.

The preconditioning operators compensate some of amplitude decays due to the geometrical spreading and radiation pattern of each parameter and it need for successful FWI to converge the correct model. There are some approaches to compensate the desensitization such as based on approximate Hessian(Prattet al., 1998: Butzer et al, 2013). These method offers a much better convergence and can estimate better imaging in subsurface than conventional gradient method as steepest decent. On the other hand, the disadvantage of these method is the burden of high computational cost because it requires many forward calculation to build the Hessian matrix.

In this study, we suggest a new preconditioning gradient method that seeks preconditioning operator by scattering theory instead of many forward calculations. The purpose of this method is to get the better imaging results than conventional method without extra computational cost. The procedure of building the preconditioning operator is similar with approximate Hessian method(Butzer et al., 2013). Firstly, calculation of sensitivity $\left(\frac{\partial u_{i}\left(\mathbf{x}_{r}\right)}{\partial m(\mathbf{x})}\right)$ using the scattering theory and building the preconditioning operator as (4)

$$
H_{m}(\mathbf{x})=\sum_{s} \sum_{r} \frac{\partial u_{i}\left(\mathbf{x}_{r}\right)}{\partial m(\mathbf{x})} \frac{\partial u_{i}\left(\mathbf{x}_{r}\right)}{\partial m(\mathbf{x})}
$$

\section{INVERSION RESULTS}

\section{1 single anomaly model}

3.1.1 inversion settings

We conduct numerical experiments to compare the results obtained by the new method with those from a conventional method (Tarantola, 1984). Firstly, a single anomaly model with one anomalous block is used to confirm the performance of the new method. We implement a 2D numerical simulation in frequency domain and seek scattering angle in algorithm to apply the scattering theory to make the preconditioning operator of each parameter. Figure 1 shows a true model and table 1 shows model parameter settings. The acquisition geometry consists of 100 sources spaced every $20 \mathrm{~m}$ and recorded by 200 receivers spaced every $10 \mathrm{~m}$. Initial models are homogeneous model using the value of background in each parameter. In this model, we used 100 iterations and frequencies were ranging from $7.0 \mathrm{~Hz}$ to $11.5 \mathrm{~Hz}$ every $0.5 \mathrm{~Hz}$.

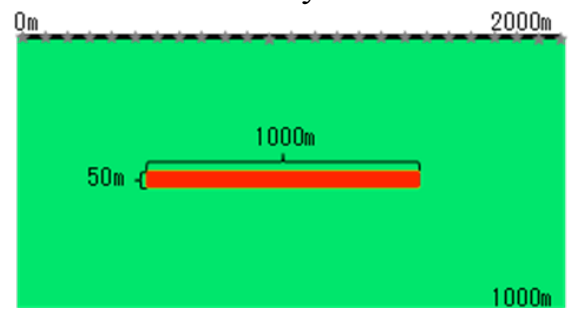

Figure 1. True model and acquisition geometry

Table 1. model parameter settings

\begin{tabular}{|l|c|c|l|}
\hline parameter & $\boldsymbol{\lambda}$ & $\boldsymbol{\mu}$ & \multicolumn{1}{c|}{ density } \\
\hline & $\mathrm{Gpa}$ & $\mathrm{Gpa}$ & $\mathrm{Kg} / \mathrm{m}^{\wedge} 3$ \\
\hline background & 4.00 & 4.00 & 2300 \\
\hline anomaly & 4.12 & 4.12 & 2250 \\
\hline
\end{tabular}

\subsection{2 results}

Figure2(a), (b), (c) show the results obtained by conventional method and each figure represents the inversion result of $\lambda, \mu$ and density, respectively. Figure2(d),(e),(f) show the results obtained by new method proposed by this study. In single anomaly model, the results obtained by conventional method are captured local minima and they cannot be estimated accurate value. On the other hand, the results obtained by new preconditioning method can be estimated reliable value in all parameter including density. These results indicate the results of the new method are more closed to true model than conventional one. Moreover, convergence of misfit function is faster and its value is smaller than 

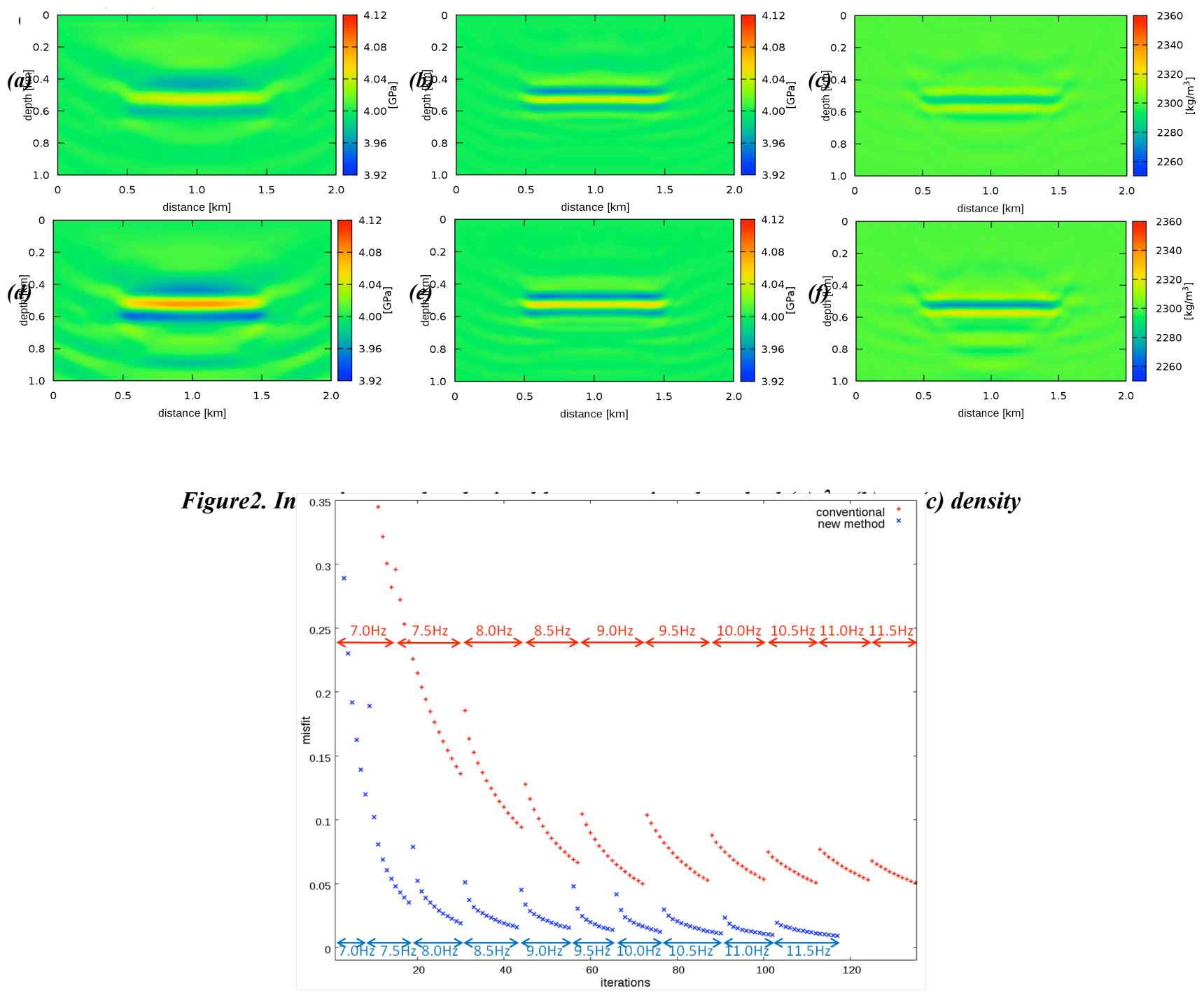

Figure 3. misfit function: red symbol represents the result of conventional method and blue symbol represents the result of new method

\section{2 cross talk model}

\subsection{1 inversion settings}

Secondly, a model with two anomalous blocks with different anomalous values are used to confirm the performance of the crosstalk among the kinematic parameters. Figure 4 shows a true model and table 2 shows model parameter settings.
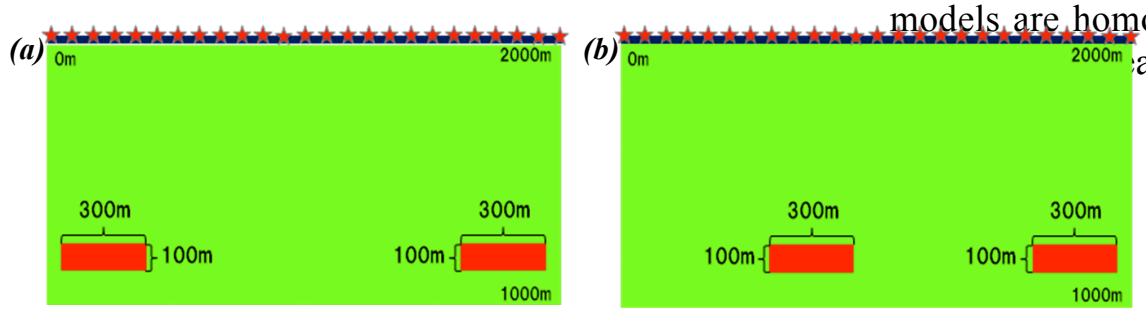

The acquisition geometry are same with single anomaly model, that consists of 100 sources spaced every $20 \mathrm{~m}$ and recorded by 200 receivers spaced every $10 \mathrm{~m}$. The anomalous blocks of each parameter has $2 \%$ higher than background values. Initial models are homogeneous model using the value of

Figure 4. True model and acquisition geometry (a) $\lambda$, (b) $\mu$, (c) density

Table2. model parameter settings

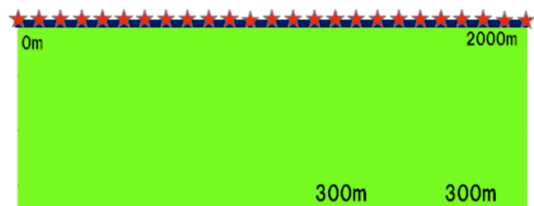

\begin{tabular}{|l|c|c|l|}
\hline parameter & $\boldsymbol{\lambda}$ & $\boldsymbol{\mu}$ & density \\
\hline & $\mathrm{Gpa}$ & $\mathrm{Gpa}$ & $\mathrm{Kg} / \mathrm{m}^{\wedge} 3$ \\
\hline background & 4.00 & 4.00 & 2300 \\
\hline anomaly & 4.08 & 4.08 & 2346 \\
\hline
\end{tabular}



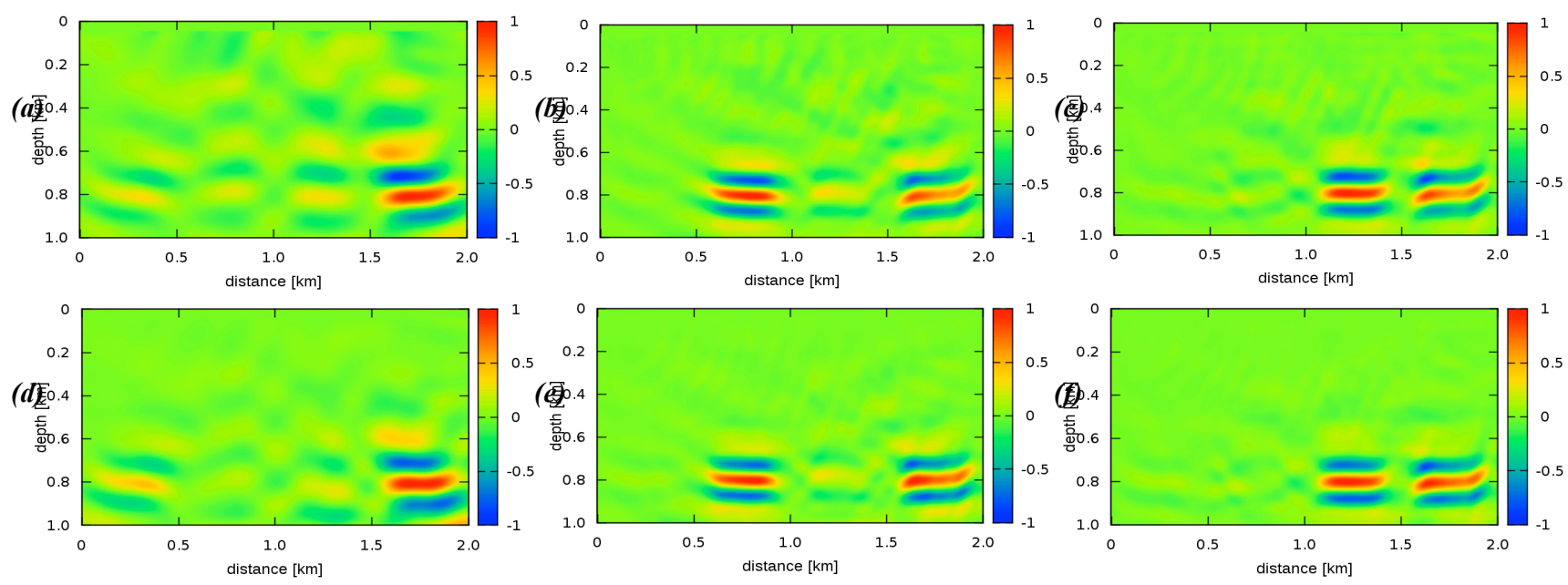

Figure5. Normalized Inversion results obtained by conventional method (a) $\lambda$, (b) $\mu$, (c) density

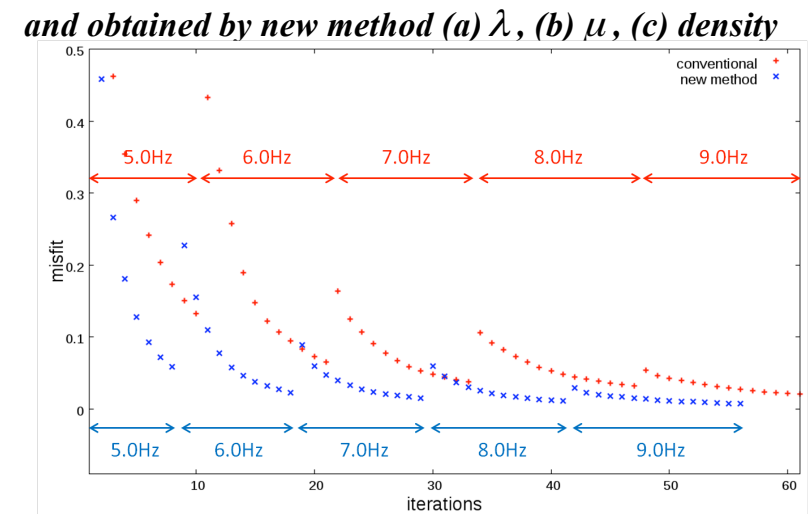

Figure 6. misfit function: red symbol represents the result of conventional method and blue symbol represents the result of new method

\subsection{2 inversion results}

Comparing with the degree of crosstalk, normalized inversion results are shown in Figure 5. These results are normalized the difference from the initial model of each parameter. Figure5(a), (b), (c) show the results obtained by conventional method. Each figure shows $\lambda, \mu$ and density results, respectively. Figure5(d), (e), (f) show the results obtained by new method. Each figure shows $\lambda, \mu$ and density results, respectively. In figure 5 (a), left side of $\lambda$ anomaly estimate ambiguous image and there are false images in the place of $\mu$ and density at the same level with left side of $\lambda$ anomaly. In contrast, left side of $\lambda$ anomaly image are clearer than false images in figure5 (d). In figure 5(b), two $\mu$ anomalies are estimated appropriate place but horizontal resolution are poorer than the results of $\mu$ in figure 5(e). Moreover, false image in place of density are mitigated in figure5(e) compared with figure5(b). Comparing with results of figure5(c) and (f), the resolution of right side anomaly of density in figure 5(f) is higher and false image in the place of $\mu$ are mitigated. The convergence of the misfit function and the misfit behaved optimized as among the kinematic parameters was lowered than

in the conventional FWI. All results show that our new method would estimate the desired parameter values and minimize the cross-talk.

\section{CONCLUSION}

Our study presents the new preconditioning gradient method for FWI based on elastic scattering theory instead of many forward calculations to make the preconditioning operator of each parameter and numerical results show the effectiveness of our method. This method can estimate more reliable results than conventional one and restrain the rise of computational costs.

\section{REFERENCES}

1)Virieux, J., Operto, S., 2009, An overview of full-waveform inversion in exploration geophysics, Geophysics, 74, WCC1-WCC26.

2)Gardner, G., L. Gardner, and A. Gregory, 1974, Formation velocity and density - The diagnostics basics for stratigraphic traps: Geophysics, 39, 770-780

3)Tarantola, A. 1984. Inversion of seismic reflection data in acoustic approximation, Geophysics, 49, 1259-1266. 
4)Pratt, R. G., Shin, C., \& Hicks, G. J., 1998, Gauss-Newton and full Newton methods in frequency-space seismic waveform inversion, Geophysics, 133, 341-362.

5)Butzer, S., Kurzmann, K., \& Bohlen, T. 2013, Applying a diagonal Hessian approximation for preconditioning in 3D elastic full waveform inversion, 17, 193-204. The Wave Inversion Technology Consortium. 